\title{
Magnetic resonance spectroscopy - its added value in brain glioma multiparametric assessment
}

\author{
Rudnay $\mathrm{M}^{1}$, Waczulikova $\mathrm{I}^{2}$, Bullova $\mathrm{A}^{3}$, Rjaskova $\mathrm{G}^{4}$, Chorvath $\mathrm{M}^{5}$, Jezberova $\mathrm{M}^{6}$, Lehotska $\mathrm{V}^{1}$ \\ IInd Department of Radiology, Faculty of Medicine of Comenius University in Bratislava and St. Elizabeth's Cancer Institute, \\ Bratislava, Slovakia. viera.lehotska@ousa.sk
}

\begin{abstract}
OBJECTIVE: In this study, we analysed the results of magnetic resonance spectroscopy (MRS) in the patients with gliomas, including the error rate, MRS parameters variability, correlations with gene mutations and overall usefulness for clinical practice.

MATERIAL AND METHODS: Eighty patients with glial tumours were examined by multiparametric MRI completed with single voxel MRS, as one group, then as two separate groups according to progression of the disease after the initial surgery. The error rate between the groups, MRS parameters variability, hazard ratios and correlations between metabolites, genetic markers and tumour grade were all analysed.

RESULTS: Variability in $\mathrm{Cho} / \mathrm{Cr}(\mathrm{h})$ was significantly higher in the group with a disease progression $(\mathrm{p}=$ 0.044). In the patients with a stable disease, strong significant negative correlations between $\mathrm{Cho} / \mathrm{Cr}$ and Cho/NAA with p53 mutation $(-0.945$ and -0.812 respectively, $\mathrm{p}<0.05)$ and between $\mathrm{Cho/Cr}$ and IDH1, 2 mutation $(-0.796, p<0.05)$ were found. In the patients with tumour progression, a significant positive correlation of NAA/Cr with $1 \mathrm{p} 19 \mathrm{q}$ codeletion $(0.486, \mathrm{p}<0.05)$ and of $\mathrm{Cho} / \mathrm{Cr}$ and Cho/NAA values with p53 mutation $(0.477$ and $0.416, \mathrm{p}<0.05)$ were identified. Tumour grade positively correlated with $\mathrm{Cho} / \mathrm{Cr}$ values $(0.304, p=0.02)$ in the whole patient group.

CONCLUSION: MRS brings an added value to multiparametric MRI evaluation of brain tumours in the patient follow-up after an initial surgery, especially in ambiguous findings (Tab. 5, Fig. 2, Ref. 29). Text in PDF www.elis.sk

KEY WORDS: glioma, MR spectroscopy, single voxel spectroscopy, choline, N-acetyl aspartate, creatine.
\end{abstract}

\section{Introduction}

Primary glial tumours of the central nervous system (CNS) are a large and diverse group of tumours with a basic common feature - origin in intracranial tissues. As with cancers of other systems, CNS tumours have differing degrees of malignancy and associated prognosis $(1,2)$.

Glial tumours represent the most common category of intraaxial brain tumours. Individual tumour types differ in predilection

${ }^{1}$ IInd Department of Radiology, Faculty of Medicine of Comenius University in Bratislava and St. Elizabeth's Cancer Institute, Bratislava, Slovakia, ${ }^{2}$ Department of Biophysics, Faculty of Mathematics, Physics and Informatics od Comenius University, Bratislava, Slovakia, ${ }^{3}$ Department of Neurology, St. Michael University Hospital, Bratislava, Slovakia, ${ }^{4}$ Department of Radiology and Medical Imaging, L. Pasteur University Hospital, Košice, Slovakia, ${ }^{5}$ Department of Radiation Oncology, St. Elizabeth's Cancer Institute, Bratislava, Slovakia, and ${ }^{6}$ Dr. Magnet L.t.d., Bratislava, Slovakia

Address for correspondence: V. Lehotská, Assoc Prof, MD, PhD, IInd Department of Radiology, Faculty of Medicine of Comenius University in Bratislava and St. Elizabeth's Cancer Institute, Bratislava, Heydukova 10, SK-812 50 Bratislava, Slovakia.

Phone: +421.2 .32249178$

Acknowledgement: This work was partially supported by the grant KEGA 041UK4/2020 from the Slovak Ministry of Education, Science, Research and Sport. for location and age, in their appearance in imaging and in histological characteristics. Since 2016, the 4th WHO classification significantly reflects the genetic/mutational analysis of individual tumours $(1,3)$.

Data on the incidence of gliomas varies, with studies reporting a proportion of about $30-40 \%$ of all the intracranial tumours. According to US studies, the incidence of gliomas is about 10 cases per 100,000 population, with men predominating over women 6:4. According to the National Cancer Registry of the Slovak Republic, between 1991 and 2011, the standardized incidence of brain malignancies was approximately $4-8.3$ cases per 100,000 population in men, with values ranging between $3.5-5.5$ per 100,000 in women. In case of the Czech Republic, the incidence of CNS cancer in 2018 was 7.18 cases per 100,000 population with a gradual increase from the year 1977. Newer data shows that CNS cancer incidence increased globally by $17.3 \%$ between 1990 and 2016 . 330000 new cases of CNS cancer were diagnosed in 2016 around the world. The incidence of age-related gliomas peaks between the ages of 40 and $65(4,5,6,28,29)$.

Only a small fraction of gliomas can be considered hereditary (about 1-5\%). Accidental occurrence with an unknown primary cause predominates. A possible role of hormonal regulation for glioma development is being studied as well. Due to the increasing number of examinations using ionizing radiation during the 
lifetime of the patients, the only known risk factor comes to the fore - ionizing radiation, for which the relative risk is reported to be 22 . Surgery, radio- and chemotherapy are the main treatment options $(4,7,8)$.

From a pathological point of view, the microscopic morphology of the tumour tissue has long dominated in the classification. In 2016, the 4th WHO classification of CNS tumours was published. This classification is based on a combination of the morphological and genetic characteristics. Based on genetic parameters, diffuse astrocytomas and oligodendrogliomas have been included in one group, differing from other forms with a limited growth mainly by prognosis. By examination of specific genetic markers - ATRX loss, IDH1/2 mutation and $1 \mathrm{p} / 19 \mathrm{q}$ codeletion a more accurate diagnosis and, mainly, a more accurate prognosis of individual tumour types is possible. A group of diffuse midline gliomas with a $\mathrm{K} 27 \mathrm{~N}$ mutation in the histone $\mathrm{H} 3$ gene was also created $(3,9,10)$.

Imaging methods, especially magnetic resonance imaging (MRI), represent the golden standard in diagnostic examination of the patients with brain tumour. In addition, MRI also represents the basis for planning of further treatment, including biopsy or surgery. The essential part is a standardized protocol created with the regard to the needs of diagnostics or multidisciplinary team, or clinical studies $(11,12)$.

In addition to standard anatomical native and post-contrast magnetic resonance imaging, the importance of multiparametric imaging with additional modalities comes to the fore. Regarding the occurrence of low-grade gliomas, it offers significant possibilities to capture the changes in nature in a more timely and accurate manner in terms of tumour dedifferentiation to a more aggressive grade or the possibility to correlate the finding of MR examination with the genetic profile (mutation analysis) of the tumour. One of the advanced modalities is MR spectroscopy (MRS), which allows, at least to some degree, in-vivo monitoring of chemical changes in tissue. Regarding the principle and possibilities of MRS, it is necessary to verify the benefits and possibilities of MRS in our practice $(11,13)$.

\section{Aim of the study}

The aim of the study was:

- To analyse the obtained spectroscopic data in the patients with a confirmed brain tumour and correlate these data with the clinical course of the disease and with histological grading, as well as with the available data on the genetic profile of individual tumours.

- To determine the degree of statistical significance of individual correlations, the degree of variability in relation to the overall clinical course of the disease, to analyse the degree of risk ratio and their statistical significance.

- To evaluate the advantages of spectroscopic data in the follow-up of the patients from the obtained data and to evaluate possible influencing factors, as well as to specify which specific parameters of MRS are the most beneficial in our practice.

\section{Patients and methods}

The initial group of patients was a group of 162 patients with verified brain tumour monitored by the brain tumours multidisciplinary team in the period from 2016 to 2020 . The only inclusion criterion was the diagnosis code $\mathrm{C} 71$ according to the 10th edition of the International Classification of Diseases (ICD-10). After the exclusion of the patients with insufficient clinical data (lost documentation) or unavailable or incomplete examinations (absence of spectroscopic examinations), we obtained the final group of 80 patients. In 78 patients it was possible to evaluate the clinical course and outcome of the disease. Out of these, 38 patients were male $(47.5 \%)$ and 42 females $(52.5 \%)$. Patients aged from 16 to 68 years, with the median of 38 years.

\section{Genetic factors}

Within the histological parameters, we also monitored the available findings within the genetic analysis. There was no uniform data set available throughout the whole group. We collected the following sum of genetic parameters in individual patients: IDH1,2, ATRX, 1p19q, p53, MGMT, BRAF, oligo2.

\section{Imaging}

As part of the follow-up, patients were examined by MRI with 3T magnetic induction device using the standard BGBT protocol, which includes the standard anatomical evaluation in $\mathrm{T} 1 \mathrm{w}$ and $\mathrm{T} 2 \mathrm{w}$ images, diffusion weighted imaging, post-contrast imaging, and MR spectroscopic examination, perfusion.

A specific protocol consists of the following sequences:

- Localizer

- 3D sequence in T2 weighing with free fluid signal suppression (FLAIR)

- T2 weighted FLAIR sequence in the transverse plane

- T1 weighted sequence in the transverse plane

- T2 weighted sequence in the transverse plane

- T2 weighted sequence in the coronary plane

- Diffusion-weighted imaging (DWI) with apparent diffusion coefficient (ADC)

- Susceptibility weighted imaging

- Post-contrast T1 weighted 3D sequence

- MR spectroscopy

- Perfusion

Within MRS, the single voxel technique (SVS) ${ }^{1} \mathrm{H}$-spectroscopy ( $\left.{ }^{1} \mathrm{H}-\mathrm{MRS}\right)$ using a PRESS sequence with a long TE time was used. The position of the ROI was determined by the examiner based on a multiplanar anatomical view.

The resulting spectral analysis of the obtained curve was performed directly by dedicated software tool within the scanner, while the monitored metabolites were $\mathrm{N}$-acetyl aspartate (NAA), choline (Cho) and creatine $(\mathrm{Cr})$. The results were recorded as a dimensionless numerical value of the ratio of individual metabolites, specifically: NAA/Cr, Cho/Cr. NAA/Cho, Cho/NAA.

For each of the ratios the values were calculated from the area under the curve (AUC), as well as from the height of the individual 


\section{8-714}

peaks of the spectral curve - marked as "(h)". For each patient, we recorded separate individual metabolite ratio values along with the date of each specific examination.

In terms of accuracy, we monitored the automatic evaluation of the curve by scanner dedicated software, which automatically indicates values with insufficient separation from noise.

For most of the patients, the results of PET examinations performed parallel with MR examinations were also available.

\section{Statistical analysis}

Statistical analysis was performed using Microsoft Excel 2016 and StatsDirect 3.3.4 software and Statistica 13.1 software. Twosample t-test or alternatively Mann-Whitney test were used to test the between-group differences. Categorical variables are presented as counts and relative frequencies and differences between two categorical variables were tested by chi-square or binomial tests. Simple bivariate correlations (Pearson's parametric or Spearman's nonparametric) for all pair-wise combinations of the variables were computed to indicate the existence and degree of mutual relationships. The estimated effect sizes in the form of hazard ratios (HR) are presented along with the respective $95 \%$ confidence intervals $(95 \% \mathrm{CI})$.

\section{Results:}

In terms of MRS data, we compared three groups of the results in patients:

- A group of patients with a stabilized disease

- A group of patients with disease progression during the study follow-up period (2016-2020)

- A group of patients with disease progression already confirmed before the study follow-up period

We monitored the number of measured values, which the dedicated system evaluated as ambiguous or potentially skewed, out of a total of 8 metabolic ratios ( 4 by AUC, 4 by height of the peaks).

In terms of one-way analysis of the variation between groups, there was no significant difference in the mean rate of inaccurate measurements among the groups $(p=0.996)$. The Bartlett's chisquare test did not reveal deviation from the assumption of equality of variance, so we can assume that the error rate distribution was similar in all the three groups.

To identify the predictive potential of MRS variables we have used Cox regression to analyse their effect on the patient progres-

Tab. 1. Hazard ratio for individual metabolic ratios, with $95 \%$ CI and $p$ values.

\begin{tabular}{lccc}
\hline Metabolites & HR & $95 \%$ CI & p \\
\hline NAA/Cr & 0.797 & 0.478 to 1.328 & $=0.3839$ \\
NAA/Cr(h) & 0.673 & 0.329 to 1.376 & $=0.2782$ \\
Cho/Cr & 1.205 & 0.919 to 1.580 & $=0.1781$ \\
Cho/Cr(h) & 1.194 & 0.777 to 1.836 & $=0.4193$ \\
NAA/Cho & 0.688 & 0.353 to 1.342 & $=0.2724$ \\
NAA/Cho(h) & 0.666 & 0.325 to 1.367 & $=0.2676$ \\
Cho/NAA & 0.932 & 0.793 to 1.096 & $=0.3951$ \\
Cho/NAA(h) & 0.890 & 0.723 to 1.095 & $=0.2960$ \\
\hline
\end{tabular}

Tab. 2. Mann-Whitney test p-values for metabolites ratios variability.

\begin{tabular}{llcl}
\hline $\mathrm{NAA} / \mathrm{Cr}$ & 0.3146 & NAA/Cho & 0.5905 \\
$\mathrm{NAA} / \mathrm{Cr}(\mathrm{h})$ & 0.5380 & NAA/Cho(h) & 0.9161 \\
$\mathrm{Cho} / \mathrm{Cr}$ & 0.1691 & Cho/NAA & $\mathbf{0 . 1 0 4 7}$ \\
Cho/Cr(h) & $\mathbf{0 . 0 4 3 9}$ & Cho/NAA(h) & 0.2885 \\
\hline
\end{tabular}

sion-free survival after adjusting for age and sex. Due to differences in follow-up patient scheduling and in the pattern of missed or re-scheduled appointments that depended, among others, on the availability of instrumentation, we included these variables as aggregated patient characteristics (averaged over all examinations). The results are presented in Table 1.

The results were not statistically significant at the level of significance of 0.05 , however, for the variable $\mathrm{Cho} / \mathrm{Cr}$ the achieved level of significance was less than 0.2 , which allowed including this variable into a model with multiple predictors. Both Cho/ $\mathrm{Cr}$ metabolic ratios showed an increased hazard of progression with each unit of increase in the variable. On the contrary, other metabolic ratios were associated with a statistically insignificant reduction in hazard ratio.

To compare the between-group variability in the repeated measurements of the MRS variables, we analysed the coefficients of variation computed from individual data by Mann-Whitney test. These results indicated that most of the metabolites studied did not show a statistically significant difference in the variability between the groups of patients with progression and patients with a stable disease. The exceptions were the values of the $\mathrm{Cho} / \mathrm{Cr}(\mathrm{h})$ ratio, which showed a statistically significant difference when compared by the Mann-Whitney test, as well as by alternative nonparametric tests (Flinger-Policell test and Brunner-Munzel

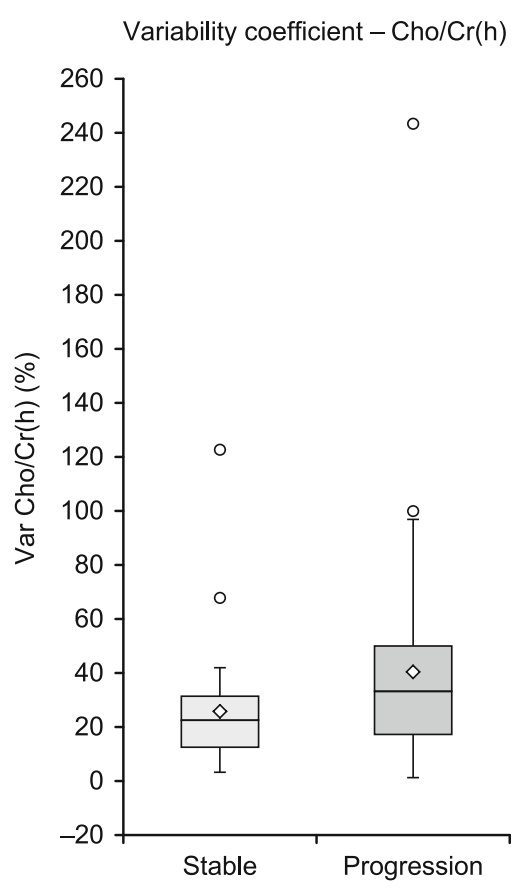

Fig. 1. Variability of $\mathrm{Cho} / \mathrm{Cr}(\mathrm{h})$ between the two groups of patients. 


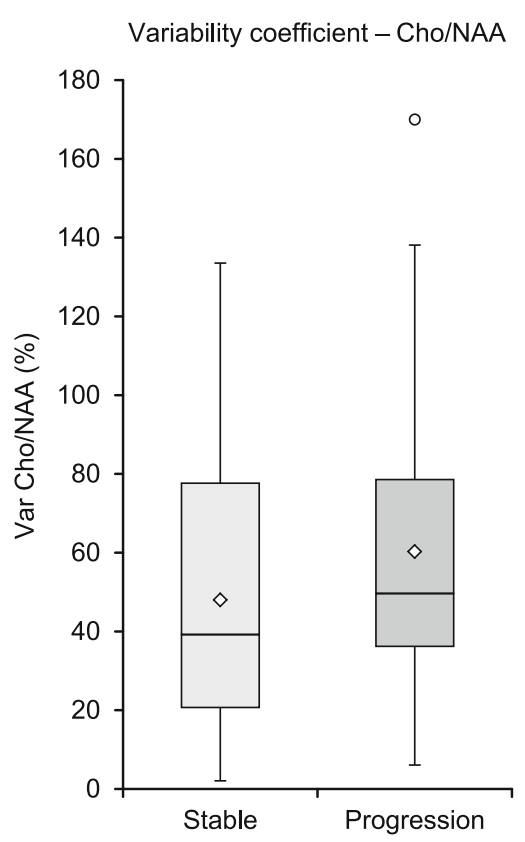

Fig. 2. Variability of Cho/NAA between the two groups of patients.

test). In the case of Cho/NAA ratio, the values reached the borderline value of statistical significance - in patients with progression the variability could be higher, $p$ values are almost identical for Mann-Whitney test as well as Flinger-Policell test and BrunnerMunzel test ( 0.1047 and 0.1039 and 0.1080 , respectively). All p values according to Mann-Whitney test are in Table 2. Box and whiskers plots for variability of $\mathrm{Cho} / \mathrm{Cr}(\mathrm{h})$ and $\mathrm{Cho} / \mathrm{NAA}$ ratios are presented in Figures 1 and 2.

In the matter of correlation, we performed an analysis between the aggregate values of individual metabolic conditions and individual available genetic markers, and we also analysed the grade of tumours by calculation of Pearson correlation coefficient with pairwise principle. The analysis was done for the whole group of patients and for subgroups by progression.

We also made correlations between the two types of calculation of metabolic ratios, according to the area under the curve versus the height of the peaks. In the whole group of patients, we found a significant correlation mainly in NAA/Cho and NAA/Cho(h): 0.905 and Cho/NAA and Cho/NAA(h): 0.821. The least significant correlation was for the ratios $\mathrm{Cho} / \mathrm{Cr}$ and $\mathrm{Cho} / \mathrm{Cr}(\mathrm{h}): 0.726$. All correlations were statistically significant $(\mathrm{p}<0.05)$.

Regarding correlations between genetic markers and median of the values of metabolites ratios, in case of the whole group of patients we found the following results: In the case of the ATRX mutation, we observed a slight positive correlation in the case of $\mathrm{NAA} / \mathrm{Cr}$ and $\mathrm{NAA} / \mathrm{Cr}(\mathrm{h}): 0.553$ and 0.473 respectively, as well as with NAA/Cho and NAA/Cho(h) ratios: 0.504 and 0.425 respectively, but these did not reach statistical significance. However, we observed a significant negative correlation with the metabolites ratio Cho/NAA, which reached a statistical significance: $-0.825, \mathrm{p}<0.05$. In the case of the p53 mutation, we observed a slight negative correlation in the case of the ratios NAA/Cr, NAA/ $\mathrm{Cr}(\mathrm{h}):-0.248$ and -0.304 that did not reach statistical significance as well as a moderate negative correlation with NAA/Cho and NAAC/Cho(h), which reached a statistical significance: -0.380 and $-0.419(\mathrm{p}<0.05)$

In the group of patients with a stabilized disease status, we found a strong significant correlation of IDH2 mutation with the median value of $\mathrm{Cho} / \mathrm{Cr}(\mathrm{h})$ ratio: $-0,796, \mathrm{p}<0.05$. In the case of the p53 mutation, we observed the most significant correlations in this group - there was a significant negative correlation with the ratios $\mathrm{Cho} / \mathrm{Cr}$ and $\mathrm{Cho} / \mathrm{Cr}(\mathrm{h})$ including $\mathrm{Cho} / \mathrm{NAA}(\mathrm{h})$, reaching statistical significance: $-0.945,-0.960$ and -0.812 respectively, $\mathrm{p}<0.05$.

In the group of patients with progression of the disease we found: In the case of ATRX, a statistically significant negative correlation with Cho/NAA dominated: $-0.822(\mathrm{p}<0.05)$. In the case of the $1 \mathrm{p} 19 \mathrm{q}$ codeletion, a statistically significant correlation of $\mathrm{NAA} / \mathrm{Cr}$ and NAA/Cr(h): 0.485 and $0.456(\mathrm{p}<0.05)$ was observed. In the case of the p53 mutation, there were statistically significant

Tab. 3. Pearson's correlation index for metabolic ratios and individual genetic markers in all 3 groups - marked correlations were statistically significant.

\begin{tabular}{|c|c|c|c|}
\hline Metabolic ratio & Correlation with & $\begin{array}{c}\text { Pearson's } \\
\text { correlation index }\end{array}$ & $\mathrm{p}$ \\
\hline \multicolumn{4}{|l|}{ Whole group } \\
\hline $\mathrm{NAA} / \mathrm{Cr}$ & ATRX & 0.553 & $>0.05$ \\
\hline NAA/Cr(h) & ATRX & 0.473 & $>0.05$ \\
\hline NAA/Cho & ATRX & 0.504 & $>0.05$ \\
\hline NAA/Cho(h) & ATRX & 0.425 & $>0.05$ \\
\hline Cho/NAA & ATRX & -0.825 & $<0.05$ \\
\hline $\mathrm{NAA} / \mathrm{Cr}$ & p53 & -0.248 & $>0.05$ \\
\hline NAA/Cr(h) & p53 & -0.304 & $>0.05$ \\
\hline NAA/Cho & p53 & -0.380 & $<0.05$ \\
\hline NAA/Cho(h) & p53 & -0.419 & $<0.05$ \\
\hline \multicolumn{4}{|c|}{ Stabilized patients } \\
\hline Cho/Cr(h) & IDH2 & -0.796 & $<0.05$ \\
\hline $\mathrm{Cho} / \mathrm{Cr}$ & p53 & -0.945 & $<0.05$ \\
\hline Cho/Cr(h) & p53 & -0.960 & $<0.05$ \\
\hline Cho/NAA & p53 & -0.812 & $<0.05$ \\
\hline \multicolumn{4}{|c|}{ Patients with progression } \\
\hline Cho/NAA & ATRX & -0.822 & $<0.05$ \\
\hline $\mathbf{N A A} / \mathbf{C r}$ & 1p19q codeletion & 0.485 & $<0.05$ \\
\hline NAA/Cr(h) & 1p19q codeletion & 0.456 & $<0.05$ \\
\hline NAA/Cho & p53 & -0.479 & $<0.05$ \\
\hline NAA/Cho(h) & p53 & -0.486 & $<0.05$ \\
\hline Cho/Cr(h) & $\mathrm{Ki}-67$ index & 0.307 & $>0.05$ \\
\hline
\end{tabular}

Tab. 4. Spearman's correlation index for metabolic ratios and individual genetic markers in the group with disease progression - marked correlations were statistically significant.

\begin{tabular}{lccc}
\hline Metabolic ratio & Correlation with & $\begin{array}{c}\text { Spearman's } \\
\text { correlation index }\end{array}$ & $\mathrm{p}$ \\
\hline Patients with progression & & \\
\hline NAA $/$ Cr & 1p19q codeletion & $\mathbf{0 . 5 0 0}$ & $<\mathbf{0 . 0 5}$ \\
NAA $/$ Cr(h) & 1p19q codeletion & $\mathbf{0 . 5 3 9}$ & $<\mathbf{0 . 0 5}$ \\
Cho/Cr & $\mathbf{p 5 3}$ & $\mathbf{0 . 4 7 7}$ & $>\mathbf{0 . 0 5}$ \\
Cho/NAA & $\mathbf{p 5 3}$ & $\mathbf{0 . 4 1 6}$ & $>\mathbf{0 . 0 5}$ \\
Cho $/$ NAA $($ h) & $\mathbf{p 5 3}$ & $\mathbf{0 . 4 1 6}$ & $>\mathbf{0 . 0 5}$ \\
NAA $/$ Cho(h) & $\mathbf{p 5 3}$ & $\mathbf{- 0 . 4 1 7}$ & $>\mathbf{0 . 0 5}$ \\
\hline
\end{tabular}


Tab. 5. Pearson's correlation index for metabolic ratios and grade of tumours in the whole group - marked correlations were statistically significant.

\begin{tabular}{lcc}
\hline Metabolic ratio & Pearson's correlation index & $\mathrm{p}$ \\
\hline $\mathbf{C h o} / \mathbf{C r}$ & $\mathbf{0 . 3 0 4}$ & $\mathbf{0 . 0 2}$ \\
$\mathbf{C h o} / \mathbf{C r}(\mathbf{h})$ & $\mathbf{0 . 2 3 1}$ & $\mathbf{0 . 0 5}$ \\
\hline
\end{tabular}

negative correlations with NAA/Cho and NAA/Cho(h): -0.479 and $-0.486(\mathrm{p}<0.05)$. In the case of the Ki-67 index, the correlations did not reach statistically significant values, the most positive correlation was $\mathrm{Cho} / \mathrm{Cr}(\mathrm{h}): 0.307$. The results are shown in Table 3.

In the case of Spearman's correlation coefficient, there were fewer statistically significant $(\mathrm{p}<0.05)$ correlations, but even with this approach, the statistically significant positive correlation was achieved in case of $1 \mathrm{p} 19 \mathrm{q}$ codeletion and the median values of $\mathrm{NAA} / \mathrm{Cr}$ and NAA/Cr(h): 0.500 and 0.539 . The $\mathrm{p} 53$ mutation and $\mathrm{Cho} / \mathrm{Cr}$, Cho/NAA and Cho/NAA(h) ratios medians also achieved a statistically significant positive correlation: 0.477 and 0.416 for both Cho/NAA and Cho/NAA(h) respectively. Synchronously, there was a statistically significant negative correlation with NAA/ Cho (h): -0.417 . The results are shown in Table 4.

Regarding the tumour grade, we determined a statistical significance with the median metabolite ratio in the whole patient group, and thus a positive correlation of $\mathrm{Cho} / \mathrm{Cr}$ and $\mathrm{Cho} / \mathrm{Cr}(\mathrm{h})$ : 0.304 and 0.231 ( $\mathrm{p}=0.02$ and 0.05 , respectively) (Tab. 5).

We also compared the correlations of metabolites ratios between each other at the time of progression between the two patient groups, we found that in the case of Spearman's correlation coefficient with the pairwise principle, more significant differences were visible when comparing the individual groups, especially in the case of $\mathrm{Cho} / \mathrm{Cr}$ and $\mathrm{Cho} / \mathrm{Cr}(\mathrm{h})$ with $\mathrm{NAA} / \mathrm{Cr}$ and NAA/ $\mathrm{Cr}(\mathrm{h})$ correlations. In the group of patients with a stabilized state, moderate negative correlations were present: $-0.400-0.425$ and $-0.461-0.427$ respectively, which were statistically significant, while in the patients with progression, the same correlations were almost zero and were not statistically significant.

\section{Discussion}

MR spectroscopy has been intensively studied for several decades - regarding the technological development and also in terms of clinical application. From a general view of the metabolic tumour changes, García-Figueiras et al (14) considered as the most significant the differences or increase of the choline level, whose spectrum consists of 3 components - choline, phosphocholine and glycerophosphocholine. The difference between healthy and tumorous tissue is usually manifested by an increase in the concentration of choline. MR spectroscopy was originally created primarily for the assessment of brain tumours, but currently is also used in the diagnosis of other tumours - e.g. breast tumours, where similarly to the brain, MRS forms a part of multiparametric imaging, thus enabling monitoring of the phenotypic variability and heterogeneity of the tumours. ${ }^{1} \mathrm{H}$-spectroscopy is not the only studied application, other susceptible nuclei - like ${ }^{31} \mathrm{P}$-spectroscopy are studied as well. Application for other brain diseases, like attention deficit and hyperactivity disorder is studied too $(15,16,17,18)$.

In our study, we determined the benefits of spectroscopic findings for our practice as well as compared our findings with previously published literature on this topic.

A similar issue was addressed by Bulik et al (19), who studied the use of MRS in glioma grading. According to their results, low grade gliomas are characterized by high $\mathrm{N}$-acetylaspartate concentrations, relatively low choline levels and by the absence of lactate and lipids. The increase in creatine indicated an early progression and a malignant transformation of the tumour. In gliomas up to grade III, a progression of the tumour grade - i.e. dedifferentiation - is accompanied by a gradual decrease in the concentration of N-acetylaspartate and myoinositol and synchronously, by an increase in choline. Malignant transformation in the tumour grade III is then also accompanied by the appearance of lactate and lipids, while this is especially apparent in the grade IV - i.e. glioblastoma. Changes in the N-acetylaspartate and choline concentrations significantly correlate with the changes in the Ki-67 proliferation index and therefore can be used in the monitoring and determination of tumour areas with an aggressive growth. In our study, we found a similar conclusion, where in the group of patients with tumour progression, we observed a higher $\mathrm{HR}$ in the ratio $\mathrm{Cho} / \mathrm{Cr}$ and $\mathrm{Cho} / \mathrm{Cr}(\mathrm{h})-1.205$ (95\% CI: 0.915 to 1.580 ) and 1.194 (95\% CI: 0.777 to 1.836 ), while in the numerator metabolic ratios with NAA showed a HR less than 1. The variability analysis confirmed a statistically significant and marginally significant variability of $\mathrm{Cho} / \mathrm{Cr}(\mathrm{h})$ and $\mathrm{Cho} / \mathrm{NAA}$ values, respectively. In our correlation study, we also observed a slight positive correlation of the ratio $\mathrm{Cho} / \mathrm{Cr}$ and $\mathrm{Cho} / \mathrm{Cr}(\mathrm{h})$ and tumour grade in the whole cohort $(0.304$ and 0.231 , respectively). These values proved to be statistically significant. Also, in the group of patients with a disease progression, the ratio Cho/ $\mathrm{Cr}$ and $\mathrm{Cho} / \mathrm{Cr}(\mathrm{h})$ and tumour grade $(0.253$ and 0.233 , respectively), were without a statistical significance. A similar conclusion about metabolites in glioblastoma was found in the study of Hnilicová et al (20]. In the case of the Ki-67 index, we observed a slight positive correlation with $\mathrm{Cho} / \mathrm{Cr}(\mathrm{h})$ ratio (0.307) in the patients with progression, although the results were not statistically significant.

The correlation between choline level and Ki-67 proliferation index was also a subject of interest of the study by Shimizu et al (21). They performed a spectral analysis in 26 patients with gliomas before a surgical resection. The measurement was performed as semi-quantitative, using a standardized reference control. Ki-67 values were evaluated from surgically removed tumours. The authors also took into account the homogeneity of the voxel. As the result, they found a strong linear correlation between choline and $\mathrm{Ki}-67$ values, but only for homogeneous voxels, while no correlation was found for inhomogeneous voxels. Having a similar finding in our study, it is of high probability, that the inhomogeneity of individual voxels in SVS was the cause of insufficient correlation of choline and $\mathrm{Ki}-67$ in the patients with progression. In the case of the Pearson correlation coefficient of the whole patient group, we noted a statistical significance. 
In the study by Law et al (22), they compared the sensitivity, specificity and predictive values of MR perfusion examination and MR spectroscopy in comparison with conventional, i.e. anatomical MR imaging. The differences in perfusion as well as in the relative ratios of the metabolites $\mathrm{Cho} / \mathrm{Cr}$ and $\mathrm{Cho} / \mathrm{NAA}$ between low grade gliomas and high grade gliomas were all statistically significant. In our study, we noted a statistically significant and marginally significant increased variability in the Cho/ $\mathrm{Cr}$ ratios and the Cho/NAA ratio, respectively, as well as a significant positive correlation of $\mathrm{Cho} / \mathrm{Cr}$ and $\mathrm{Cho} / \mathrm{Cr}(\mathrm{h})$ ratios and the tumour grade.

Despite less pronounced results, a multiparametric imaging in brain tumour imaging provides some added value. The study by Fellah et al (23) stated that although in the case of oligodendroglial tumours multiparametric examination itself provided only little benefit for distinguishing the genotype of tumours in terms of the presence of $1 \mathrm{p} 19 \mathrm{q}$ deletion, it increases the overall accuracy of tumour grading. In our study, we similarly observed a statistically significant correlation of choline i.e. $\mathrm{Cho} / \mathrm{Cr}$ and $\mathrm{Cho} / \mathrm{Cr}(\mathrm{h})$ and tumour grade, and a statistically significant correlations of NAA/ $\mathrm{Cr}$ and NAA/Cr(h) in the case of the $1 \mathrm{p} 19 \mathrm{q}$ codeletion, as well.

The relationship of Cho/Cr metabolic ratio to tumour grading, the relationship of maximal normalized Cho values and mean Cho values to grading, as well as the sensitivity and specificity of examinations were documented by Senft et al (24). They similarly pointed out the importance and usefulness of decision-making on the therapeutic process. With regards to sensitivity and specificity, monitoring of the $\mathrm{Cho} / \mathrm{Cr}$ ratio proved to be the best choice. In their meta-analysis, Chuang et al (25) also found an increased accuracy in the differentiation of the tumour tissue from necrosis by using MRS, namely using $\mathrm{Cho} / \mathrm{Cr}$ and Cho/NAA values. In our study, we applied monitoring of these parameters in two variants (height / area under the curve), where we acquired a positive, statistically significant correlation of $\mathrm{Cho} / \mathrm{Cr}$ with tumour grade in the whole group of patients.

Similar results were observed by the study of Chen et al. They also showed a positive correlation of $\mathrm{Cho} / \mathrm{Cr}$ with a higher tumour grade (26). Similarly, Bulakbasi and Paksoy noted a gradual increase in $\mathrm{Cho} / \mathrm{Cr}$ values that correlated well with tumour progression (27]. In our study, we proved a statistically significant difference in the negative correlation of $\mathrm{Cho} / \mathrm{Cr}$ and $\mathrm{NAA} / \mathrm{Cr}$ in both calculations (height / area) in the group of stable patients, compared to the group with progression, where no correlation was present.

\section{Conclusion}

Despite the limitation of the study design, we achieved several conclusions for practice:

- MRS data, namely the values of the ratio $\mathrm{Cho} / \mathrm{Cr}$ and $\mathrm{Cho} / \mathrm{Cr}(\mathrm{h})$, showed a correlation with tumour grading.

- These metabolic ratios have been significantly negatively correlated with $\mathrm{NAA} / \mathrm{Cr}$ values and $\mathrm{NAA} / \mathrm{Cr}(\mathrm{h})$ values respectively in the group of patients with stable disease, while in the case of patients with tumour progression the given parameters practically did not correlate.
- We noted a correlation between the values of metabolites and several genetic markers. The most significant was the correlation of metabolites and 1p19q codeletion and p53 mutation.

- We observed a statistically significant and marginally significant variability in $\mathrm{Cho} / \mathrm{Cr}(\mathrm{h})$ and Cho / NAA ratios respectively between groups of patients with stable disease and with tumour progression.

- In the analysis of the hazard ratios, the values of $\mathrm{Cho} / \mathrm{Cr}$ and $\mathrm{Cho} / \mathrm{Cr}(\mathrm{h})$ have slightly higher $\mathrm{HR}$, although with an insufficient statistical significance.

- When comparing the degree of inaccuracy between the groups, we did not notice any significant deviations.

- Overall, we also confirmed the dominant usefulness of choline values and choline to creatine and NAA to choline ratio.

- Methods for calculating values by height and by area under the curve correlated well.

Regarding the mentioned limitations, it is appropriate to consider further standardization of tumour genetic profiling and that of individual examination scheduling, as well as to reduce the degree of variability of the sample voxel location, or to standardize the location. Homogeneity analysis using multivoxel spectroscopy could be beneficial.

\section{References}

1. McFaline-Figueroa JR, Lee EQ. Brain Tumors. Am J Med 2018; 131 (8): 874-882.

2. Mellinghoff IK, Gilbertson RJ. Brain Tumors: Challenges and Opportunities to Cure. J Clin Oncol 2017 Jul 20; 35 (21): 2343-2345.

3. Reifenberger G, Wirsching HG, Knobbe-Thomsen CB, Weller M. Advances in the molecular genetics of gliomas - implications for classification and therapy. Nat Rev Clin Oncol 2017; 14 (7): 434-452.

4. Schneider T, Mawrin C, Scherlach C, Skalej M, Firsching R. Gliomas in adults. Dtsch Arztebl Int 2010; 107 (45): 799-808.

5. Duffau H. Diffuse Low-Grade Gliomas in Adults. Cham: Springer International Publishing; 2017.

6. Národný onkologický register SR - prezentácia výstupov (cited 2021Mar6]. Available from: https://iszi.nczisk.sk/nor.sr/uvod

7. Mezencev R. Epidemiology of gliomas in women diagnosed with breast cancer supports the protective role of estrogenic exposure. Bratisl Med J 2018; 119 (8): 463-468.

8. Morshed RA, Young JS, Hervey-Jumper SL, Berger MS. The management of low-grade gliomas in adults. Journal of Neurosurgical Sciences 2019; 63 (4): 450-457.

9. Švajdler M, Rychlý B, Zámečník J, Švajdler P. Update on the 2016 WHO classification of tumors of the central nervous system - Part 1: Diffusely infiltrating gliomas. Cesk Patol 2017; 53 (1): 12-21.

10. Louis DN, Perry A, Reifenberger G, von Deimling A, FigarellaBranger D, Cavenee WK et al. The 2016 World Health Organization Classification of Tumors of the Central Nervous System: a summary. Acta Neuropathol 2016; 131 (6): 803-820.

11. Ellingson BM, Bendszus M, Boxerman J, Barboriak D, Erickson BJ, Smits $\mathbf{M}$ et al. Consensus recommendations for a standardized Brain Tumor Imaging Protocol in clinical trials. Neuro Oncol 2015; 17 (9): 1188-1198. 
708-714

12. Ellingson BM, Smits M. The Path Forward: The Standardized Brain Tumor Imaging Protocol (BTIP) for Multicenter Trials. Glioma Imaging $2019 ; 267-282$.

13. Mabray MC, Barajas RF, Cha S. Modern Brain Tumor Imaging. Brain Tumor Research and Treatment 2015; 3 (1): 8.

14. García Figueiras R, Baleato González S, Padhani AR, Oleaga L, Vilanova JC, Luna A et al. Proton magnetic resonance spectroscopy in oncology: The fingerprints of cancer? Diagnostic and Interventional Radiology 2015; 22 (1): 75-89.

15. Faghihi R, Zeinali-Rafsanjani B, Mosleh-Shirazi MA, SaeediMoghadam M, Lotfi M, Jalli R, Iravani V. Magnetic Resonance Spectroscopy and its Clinical Applications: A Review. J Med Imaging Radiat Sci 2017; 48 (3): 233-253.

16. Grech-Sollars M, Ordidge KL, Vaqas B, Davies C, Vaja V, Honeyfield $\mathbf{L}$ et al. Imaging and Tissue Biomarkers of Choline Metabolism in Diffuse Adult Glioma: 18F-Fluoromethylcholine PET/CT, Magnetic Resonance Spectroscopy, and Choline Kinase $\alpha$. Cancers (Basel) 201911 (12): 1969.

17. Husarova V, Bittsansky M, Ondrejka I, Dobrota D. Correlations of ADHD symptoms with neurometabolites measured by $1 \mathrm{H}$ magnetic resonance spectroscopy. Bratisl Lek Listy 2014; 115 (10): 635-642.

18. Hnilicova P, Richterova R, Zelenak K, Kolarovszki B, Majercikova Z, Hatok J. Noninvasive study of brain tumours metabolism using phosphorus-31 magnetic resonance spectroscopy. Bratisl Lek Listy 2020; 121 (7): 488-492.

19. Bulik M, Jancalek R, Vanicek J, Skoch A, Mechl M. Potential of MR spectroscopy for assessment of glioma grading. Clinical Neurology and Neurosurgery 2013; 115 (2): 146-153.

20. Hnilicová P, Richterová R, Kantorová E, Bittšanský M, Baranovičová E, Dobrota D. Proton MR spectroscopic imaging of human glioblastomas at 1.5 Tesla. Gen Physiol Biophys 2017 Dec; 36 (5): $531-537$.
21. Shimizu H, Kumabe T, Shirane R, Yoshimoto T. Correlation between choline level measured by proton MR spectroscopy and Ki-67 labeling index in gliomas. AJNR Am J Neuroradiol 2000; 21 (4): 659-665.

22. Law M, Yang S, Wang H, Babb JS, Johnson G, Cha S et al. Glioma grading: sensitivity, specificity, and predictive values of perfusion MR imaging and proton MR spectroscopic imaging compared with conventional MR imaging. AJNR Am J Neuroradiol 2003; 24 (10): 1989-1998.

23. Fellah S, Caudal D, De Paula AM, Dory-Lautrec P, FigarellaBranger D, Chinot $\mathbf{O}$ et al. Multimodal MR Imaging (Diffusion, Perfusion, and Spectroscopy): Is It Possible to Distinguish Oligodendroglial Tumor Grade and 1p/19q Codeletion in the Pretherapeutic Diagnosis? American Journal of Neuroradiology 2012; 34 (7): 1326-1333.

24. Senft C, Hattingen E, Pilatus U, Franz K, Schänzer A, Lanfermann $\mathrm{H}$ et al. Diagnostic value of proton magnetic resonance spectroscopy in the noninvasive grading of solid gliomas: comparison of maximum and mean choline values. Neurosurgery 2009; 65 (5): 908-913

25. Chuang MT, Liu YS, Tsai YS, Chen YC, Wang CK. Differentiating Radiation-Induced Necrosis from Recurrent Brain Tumor Using MR Perfusion and Spectroscopy: A Meta-Analysis. PLOS ONE 2016; 11 (1).

26. Chen J, Huang SL, Li T, Chen XL. In vivo research in astrocytoma cell proliferation with $1 \mathrm{H}$-magnetic resonance spectroscopy: correlation with histopathology and immunohistochemistry. Neuroradiology 2006; 48 (5): $312-318$.

27. Bulakbaşı N, Paksoy Y. Advanced imaging in adult diffusely infiltrating low-grade gliomas. Insights into Imaging 2019; 10 (1).

28. GBD 2016 Brain and Other CNS Cancer Collaborators. Global, regional, and national burden of brain and other CNS cancer, 1990-2016: a systematic analysis for the Global Burden of Disease Study 2016. Lancet Neurol 2019; 18 (4): 376-393.

29. The Institute of Health Information and Statistics of the Czech Republic (Internet] (cited 2021Mar9]. Available from: https://www.uzis.cz/

Received March 15, 2021. Accepted April 19, 2021. 